\section{DIVISION H COMMISSION 37}

\section{STAR CLUSTERS} AND ASSOCIATIONS

\author{
AMAS ET ASSOCIATIONS \\ STELLAIRES
}

\author{
Giovanni Carraro \\ Richard de Grijs \\ Bruce Elmegreen \\ Peter Stetson, \\ Barbara Anthony-Twarog, \\ Simon Goodwin, Douglas Geisler, \\ Dante Minniti
}

\title{
HIGHLIGHTS OF COMMISSION 37 SCIENCE RESULTS
}

\begin{abstract}
It is widely accepted that stars do not form in isolation but result from the fragmentation of molecular clouds, which in turn leads to star cluster formation. Over time, clusters dissolve or are destroyed by interactions with molecular clouds or tidal stripping, and their members become part of the general field population. Star clusters are thus among the basic building blocks of galaxies. In turn, star cluster populations, from young associations and open clusters to old globulars, are powerful tracers of the formation, assembly, and evolutionary history of their parent galaxies. Although their importance (e.g., in mapping out the Milky Way) had been recognised for decades, major progress in this area has only become possible in recent years, both for Galactic and extragalactic cluster populations. Star clusters are the observational foundation for stellar astrophysics and evolution, provide essential tracers of galactic structure, and are unique stellar dynamical environments. Star formation, stellar structure, stellar evolution, and stellar nucleosynthesis continue to benefit and improve tremendously from the study of these systems. Additionally, fundamental quantities such as the initial mass function can be successfully derived from modelling either the Hertzsprung-Russell diagrams or the integrated velocity structures of, respectively, resolved and unresolved clusters and cluster populations. Star cluster studies thus span the fields of Galactic and extragalactic astrophysics, while heavily affecting our detailed understanding of the process of star formation in dense environments. This report highlights science results of the last decade in the major fields covered by IAU Commission 37: Star clusters and associations. Instead of focusing on the business meeting - the out-going president presentation can be found here: http://www.sc.eso.org/ gcarraro/ splinter2015.pdf - this legacy report contains highlights of the most important scientific achievements in the Commission science area, compiled by 5 well expert members.
\end{abstract}

\section{Embedded and massive star clusters in the Milky Way}

Ignacio Negueruela, Universidad de Alicante, Spain

The past decade has been an era of discovery in the Milky Way, ushered in by our increasing capability to see through dust. This text is a short summary of the observations 
that have helped shape our understanding of massive star formation and young clusters in the Galaxy. I must apologise from the beginning for all the very relevant work that I have not been able to review. My reference list is just intended as a collection of examples to illustrate the broad range of excellent research carried out by our community. It is only natural that I concentrate on what I know best.

Deep infrared surveys of the Galactic Plane have revealed hundreds of new open clusters hidden by high obscuration. The publication of the 2MASS catalogue was followed by a number of dedicated searches, some by eye (e.g. Dutra et al. 2003), others using automated methods (e.g. Froebrich et al. 2007), that resulted in close to one thousand new cluster candidates. The Spitzer/GLIMPSE survey (Churchwell et al. 2009) presented us with a mid-IR view of the Galactic Plane at high spatial resolution, leading to a new collection of even more highly absorbed clusters (e.g. Mercer et al. 2005). The process has continued as new, deeper surveys, such as VVV, have come on line (e.g. Borissova et al. 2014; Barbá et al. 2015).

Many of these clusters are low-mass stellar groups still embedded in their parental clouds (e.g. Soares et al. 2008). Others are more massive young clusters with high-mass stars (e.g. Messineo et al. 2009; Chené et al. 2013). Finally, a significant number are simply old open clusters affected by interstellar extinction. Discriminating between these types is not always easy without more detailed investigation. Even though some semiautomated methods have been proposed to discern their nature (e.g. Beletsky et al. 2009), follow-up deep photometry (e.g. Ivanov et al. 2005) and, above all, (in most cases, nearIR) spectroscopy is necessary for a good characterisation (e.g. Zhu et al. 2009; Messineo et al. 2010). Because of this, a large number of these candidates still remain unexplored.

Infrared imaging has also resulted in a novel picture of the interstellar medium. Large numbers of bubble-like structures have been found (Churchwell et al. 2006; Simpson et al. 2012), likely marking sites of massive star formation (Deharveng et al. 2010). Highly reddened high-mass stars have been identified in the vicinity of many them (e.g. Marco \& Negueruela 2011; Pinheiro et al. 2012). In less obscured environments, bright-rimmed clouds also seem to mark sites of ongoing star formation in the immediate neightbourhood of high-mass stars (e.g. Panwar et al. 2014). Herschel observations have shown the concentration of proto-stellar cores along filaments. This increasing body of data supports a view of massive star formation as a process extending over large spatial scales, and thus resulting in measurable age spreads in many young regions (e.g. Bik et al. 2012). Infrared imaging has also allowed the identification of bow-shocks produced by high-mass stars ejected from massive clusters (e.g. Peri et al. 2012), giving rise to a lively theoretical discussion on the origin of isolated high-mass stars.

Adopting a more global point of view, statistical studies of the distribution of mid-IR sources have provided strong constraints on massive star formation in the Milky Way, providing estimates of the current global star-formation rate (e.g. Robitaille \& Whitney 2010), typical duration of different phases (e.g. Mottram et al. 2011; Morales et al. 2013) or incidence of triggered star formation (e.g. Thompson et al. 2012). More recently, and in contrast, Herschel has provided a very detailed view of gas and dust around a few massive star-formation sites (e.g. Russeil et al. 2013).

But the opportunities for discovery do not only come from infrared imaging. The combination of X-ray imaging of young clusters with IR catalogues has become the preferred method to investigate the formation of low-mass stars in environments dominated by high-mass stars (e.g. Prisinzano et al. 2011; Feigelson et al. 2013), providing valuable input for theories of high-mass star formation (e.g. Kuhn et al. 2014; Rivilla et al. 2014). Radio surveys have detected large numbers of new H II regions (e.g. Anderson et al. 2011), whose ionising stars or clusters remain in most cases still unknown. Radio 
observations have also produced one of the most significant advances in the past few years: the generalised use of geometric parallaxes to masers for determination of distances to star-forming regions, an extremely powerful tool to probe the structure of the Milky Way (see references in Reid et al. 2014).

The current view of large-scale star formation in the Milky Way, emerging from all these developments, is complex. A typical configuration may be illustrated by the G305 starforming region. High-mass stars in two moderately massive young clusters, Danks 1 and 2, drive a huge wind-blown bubble, whose rim is teeming with star-formation sites, where embedded lower-mass proto-clusters are forming just now. A diffuse population of protostars is scattered over the whole region (Hindson et al. 2013 and references). The output of such a process is a classical OB association with massive central clusters, such as Per OB1 or Cas OB8. Many giant molecular clouds seem to harbour similar configurations, among them the Carina Nebula (e.g. Preibisch et al. 2011), where Trumpler 14 has a mass $M_{\mathrm{cl}} \approx 10^{4} M_{\odot}$ (Ascenso et al. (2007)), or the W3 region (Román-Zúñiga et al. 2015) that seems to form a larger structure with W4 and W5, included within Cas OB6. Others, such as W33 (Messineo et al. 2015) or W51, lack the central massive clusters, and will very likely evolve into dispersed associations, similar to Cyg OB2 (Wright et al. 2014). This may also be the future of one of the most powerful star-forming regions known in the Milky Way, W49A (Homeier \& Alves 2005).

As a result of spatially concentrated star formation, we find massive young clusters. For many years, it was believed that the Milky Way lacked clusters with masses $\geqslant 10^{4} M_{\odot}$. Then the obscured open cluster Westerlund 1 (Wd1) was found to host a population of $>70$ supergiants of spectral types ranging from O to M (Clark et al. 2005; Negueruela et al. 2010). A direct extrapolation of the number of massive stars detected would suggests a mass $M_{\mathrm{cl}} \approx 10^{5} M_{\odot}$ for a standard initial mass function(IMF). Direct star counts in the infrared give a lower limit of $5 \times 10^{4} M_{\odot}$ (Gennaro et al. 2011). The cluster seems to have formed monolithically in a single burst lasting less than $0.4 \mathrm{Ma}$ (Kudryavtseva et al. 2012).

Many clusters have joined the list of massive clusters over the past decade. Some of them were already known, but their masses have been revised upwards. For example, Trumpler 14, mentioned above, or NGC 3603, for which Harayama et al. (2008) derive a mass in the range $M_{\mathrm{cl}}=1-1.6 \times 10^{4} M_{\odot}$ with indications of a top-heavy IMF. These mass estimates depend strongly on the distance adopted to the cluster and, hence, the extinction law. This problem becomes especially acute for the clusters close to the Galactic centre, affected by very heavy and variable reddening, with an extinction law that deviates strongly from the Galactic average (e.g. Nishiyama et al. 2009). For example, the mass of the Arches cluster is hotly debated. The extinction law adopted or even possible colour terms due to the very high reddening affect the luminosity function, which has to be later translated into a mass function. Masses ranging from $M_{\mathrm{cl}}<10^{4} M_{\odot}$ to several $10^{4} M_{\odot}$ have been found depending on assumptions (see references in Clarkson et al. 2012). But even much less reddened clusters are subject to such uncertainties. An extreme case is Westerlund 2, for which distances ranging from $\sim 3$ to $\sim 8 \mathrm{kpc}$ have been claimed (Carraro et al. 2013), because of very different estimates of the reddening law.

Besides W49A, the strongest candidate for the most massive star-forming region in the Milky Way is W43 (Bally et al. 2010; Nguyen Luong et al. 2011), located close to the point where the Galactic bar joins the Scutum-Crux arm. The vigour of star formation in this area is likely related to this location, as there are indications of inflows from the spiral arm towards the densest regions (Motte et al. (2014)). Not far from W43, and at about the same distance from the Sun, several clusters rich in red supergiants have been found (e.g. Figer et al. 2006; Davies et al. 2007). These clusters have ages in the 
10 - 20 Ma range, and estimated masses between 2 and $>5 \times 10^{4} M_{\odot}$. At least two large associations with $>10^{5} M_{\odot}$ have been found around them (Negueruela et al. 2011, 2012). The presence of so many extreme examples of present or recent star formation within a small span of the Galactic Plane $\left(\sim 6^{\circ}\right)$ seems to hint at a very privileged location. Further support for this interpretation would come from the detection of a similarly hefty complex at the opposite end of the Bar. Even though at least one massive cluster has been found in that general direction (Davies et al. 2012), the exact position where we should expect this starburst region is not certain. The young massive cluster vdBH 222 , though originally thought to mark this far end of the bar (Marco et al. 2014), seems to be closer to us (and the Galactic Centre), suggesting that very large associations do not necessarily form at special places, and so hinting at the possibility that star formation in the inner Milky Way may be rather more vigorous than in the outer spiral arms.

Finding massive young clusters is not an end in itself. They not only inform us about star formation in violent environments, but also play a significant role as laboratories for the study of high-mass star evolution. With its huge population of evolved high-mass stars spanning all stages of evolution, $\mathrm{Wd} 1$ is the prime example. The different evolutionary paths of isolated and binary high-mass stars can be directly observed and documented within its co-eval population (Clark et al. 2014 and references therein). Similarly, multiepoch spectroscopic surveys of young open clusters have become fundamental tools to understand the physical properties of high-mass stars (Evans et al. 2005, 2011, and related references). As we move into the era of large spectroscopic surveys, this potential will be fullfilled. At the same time, ALMA is starting to look at the sites of massive star formation with unprecedented resolution. In the near future, more advanced instrumentation on the 10-m class telescopes, the launch of JWST and the advent of giant telescopes with adaptive-optics-assisted instrumentation offer the promise of not only an extended era of discovery, but also profound advances in our understanding of how star formation on large scales proceeds, and how high-mass stars are born, evolve and return to the interstellar medium the enriched material that will feed the future stellar generations.

\section{Open clusters in the Milky Way}

\section{Elena Glushkova, Sternberg Observatory, Moscow, Russia}

Open star clusters (OCs) have always received special interest, because by studying these objects, it is possible to make suggestions on the structure and kinematics of the Galactic disk and its dynamical and chemical evolution. However, there are two major obstacles to achieve these goals: 1) the sampling of OCs is complete only up to distances of $800-1000 \mathrm{pc}$ and 2) no unbiased, homogeneous collection of fundamental parameters are available in the literature. Concentration of interstellar gas and dust toward the Galactic plane makes the detection of new OCs difficult especially in the visible range. To build a homogeneous catalog of OC parameters, homogeneous observational data and reliable methods of determination of the distances, ages, and reddening of the clusters are required. Nonetheless, these obstacles are cleared step by step in numerous studies, each considering few, sometimes a dozen, clusters at once.

The situation dramatically changed in the beginning of the 2000s, when large sky surveys became available. They triggered a new wave of interest to discover new, and investigate already known, open clusters. The greatest attention was drawn by TwoMicron All Sky Survey (2MASS, Skrutskie et al. 2006), whose data were collected in three near- $I R$ bands. Searches for clusters were conducted either visually, on images recorded in one of the bands, or from the Point Source Catalog using an automated 
routine (Ivanov et al. 2002; Dutra et al. 2003; Bica et al. 2003; Kronberger et al. 2006; Froebrich et al. 2007; Koposov et al. 2008; Glushkova et al. 2010; Schmeja et al. 2014; Scholz et al. 2015). They found about 2000 candidates, visible in infrared only. One thousand cluster candidates more were mined in other surveys: DENIS (Reylé \& Robin 2002), USNO-A2.0 (?), GLIMPSE (Mercer et al. 2005; Zasowski et al. 2013), UKIDSS GPS (Solin et al. 2012), VVV (Borissova et al. 2011, 2012, 2014; Solin et al. 2014), WISE (Camargo et al. 2015). Rübke et al. (2015) started the program of searching for MAssive Stars in Galactic Obscured MAssive clusterS (MASGOMAS) and developed a new automatic tool, which allows the identification of a large number of massive ( a few 1000 solar masses) cluster candidates from the $2 M A S S$ and $V V V$ catalogs.

As $2 M A S S$ is a homogeneous and all-sky catalog, many attempts were made to derive a homogeneous list of accurate physical parameter for OCs out of $J H K_{S}$ data: refer, for example, to the papers by Koposov et al. (2008), Tadross (2008, 2009, 2011), Glushkova et al. (2010), Bukowiecki et al. (2011), Kharchenko et al. (2013). However, the comparison of parameters obtained in these investigations with respect to the largest catalog by Kharchenko et al. (2013), gives mean standard deviations for the distance, age and reddening as $1.2 \mathrm{kpc}, 0.5 \mathrm{dex}$, and $0.27 \mathrm{mag}$, respectively (Netopil et al. 2015, which are definitely insufficient for comprehensive studies of the structure of the Galactic disc. Involving data from large surveys, scientists undertook massive determinations of other OC characteristics: the radial velocities of 110 OCs and the metallicities of 89 OCs were derived using data from RAdial Velocity Experiment (RAVE, Conrad et al. 2014), the mean proper motions and stellar membership probabilities for 1805 open clusters were found using UCAC4 (Dias et al. 2014).

Since open clusters provide information about the chemical pattern of Milky Way disk, the study of their chemical composition attracts special interest. Paunzen et al. (2010) compiled data from the available sources in the literature to make up the catalog of OC metallicities based on photometric data, which lists 188 clusters (Paunzen et al. 2010) and set of high-quality cluster metallicities based on high-resolution spectroscopic studies for 78 clusters (Heiter et al. 2014). Heiter et al. 2014 show that none of the current models are able to satisfactorily describe the OC's metallicity as function of galactocentric distance. The present high-resolution spectroscopic Galactic surveys include OCs among their targets: Apache Point Observatory Galactic Evolution Experiment (APOGEE, the Gaia-ESO Survey (GES, Gilmore et al. 2012), the GALactic Archaeology with HERMES (GALAH, Anguiano et al. 2014) supply accurate radial velocities and detailed chemical abundances. Using 100 OCs from the uniformly observed complete SDSS-III/APOGEE-1 DR12 dataset (OCCAM survey), Frinchaboy et al. 2015 presented age and multi-element abundance gradients for the disk of Milky Way. The INfrared Survey of Young Nebulous Clusters (IN-SYNC, Covey et al. 2015) leverages the stability and multiplex capability of the APOGEE spectrograph to obtain high resolution spectra at near-infrared wavelengths and to study the dynamics and star formation history of young clusters. Four young stellar groups in Perseus and Orion molecular complexes were investigated (Covey et al. 2015) based on homogeneous stellar parameters derived from APOGEE spectra for thousands of pre-main sequence stars (Cottaar et al. 2014). OCs are also included in the list for the space missions Gaia and Kepler.

Some projects were delivered especially to investigate OCs and derive their physical parameters in a precise and homogeneous way: for example, within the framework of Bologna Open Clusters Chemical Evolution project (BOCCE, Bragaglia \& Tosi 2006) about 50 clusters were studied. BOCCE uses both comparison between observed color-magnitude diagrams (CMDs) and stellar evolutionary models, and the analysis of high-resolution spectra to derive age, distance and chemical composition. The Open 
Clusters Chemical Abundances from Spanish Observatories survey (OCCASO, Casamiquela et al. 2014) plans to derive abundances for more than 20 chemical species in at least 6 Red Clump stars in 30 northern OCs. Nine clusters have been studied by OCCASO. Sejong Open Cluster Survey (SOS, Sung et al. 2013) - a project dedicated to providing homogeneous photometry of a large number of OCs in the SAAO Johnson Cousins' $U B V I$ system, has many relatively small sparse unstudied clusters among its targets. The OPD survey (Caetano et al. 2015) is UBVRI photometric survey of southern open OCs, which is complementary to $O A N-S P M U B V R I$ survey of 406 northern clusters (Michel et al. (2016)). Lasting 17 years, WIYN Open Cluster Study (WOCS, Mathieu \& WOCS Collaboration 2013) is dedicated to comprehensive photometric, astrometric, and spectroscopic studies of select OCs. In their recent investigation (Thompson et al. 2014) new deep wide-field optical and near-infrared photometry $\left(U B V R J H K_{S}\right)$ of the cluster M35 is presented, against which several isochrone systems are compared: Padova, PARSEC, Dartmouth, and $Y^{2}$. Two different atmosphere models are applied to each isochrone: ATLAS9 and BT-Setti. For any isochrone set and atmosphere model, observed data are accurately reproduced for all stars more massive than $0.7 M_{\odot}$. For less massive stars, Padova and PARSEC isochrones consistently produce higher temperature than observed. Dartmouth and $Y^{2}$ isochrones with BT-Setti atmospheres reproduce optical data accurately; however, they appear too blue in IR colors. WIYN's extension the Southern Open Cluster Study (SOCS, Kinemuchi et al. 2010) includes 24 clusters, wide-field photometry results are already available for three of them. Faria et al. (2014) determined the radial velocity of stars belonging to a group of open clusters using spectra with spectral resolution of 4000 and plan to calculate the mean radial velocities of a number of OCs. To investigate star formation processes, Lim et al. (2015a) initiated a photometric survey of young open clusters in the Galaxy and already studied 13 famous OCs having a wide range of surface densities $\left(\log (\sigma)=-1-3\right.$ stars $\left./ p c^{2}\right)$ and total masses $\left(500-50000 M_{\odot}\right)$ and also distributed in five different spiral arms in the Galaxy. Lim et al. (2015a) found that the slope of the IMFs in the high-mass regime appears to be shallow for massive compact clusters, and the mass of the most massive star in a given cluster also has a tendency to be large in massive clusters. Costa et al. (2015) started a program that determines the properties of Local (Orion) spiral arm. They plan to carry on a comprehensive study of 29 young OCs which includes a $U B V R I$ photometric analysis and determination of their kinematics. The first cluster NGC 2302 has already been investigated.

When large catalogs and data sets became available in the literature, and massive determination of parameters of clusters became possible, numerous automated and semiautomated techniques were developed to retrieve these parameters primarily by using color-magnitude diagrams. One of the most powerful tools is the Automated Stellar Cluster Analysis package (ASteCA, Perren et al. 2015, which makes use of positional and photometric data to provide accurate estimates of the cluster's metallicity, age, extinction and distance values, and robust stellar cluster image and photometry simulation package MASSCLEAN (Popescu \& Hanson 2010), which creates synthetic clusters and generate CMD templates for a variety of cluster masses and ages, and which mimic the observational photometric errors when using isochrone fitting (Popescu et al. 2014).

Thanks to All Sky Automated Survey (ASAS, Pojmanski et al. 2005), a large number of new Galactic Cepheids have been discovered during the last dozen years. That is why new attempts were undertaken to find Cepheids attributed to open star clusters. Taking into account all possible characteristics of these variable stars and OCs, Anderson et al. (2013) found five new genetic relations between Cepheids and clusters, and Chen et al. (2015) reported 8 new Chepheid-cluster pairs. Some papers were devoted to photometric and 
spectroscopic observations of known clusters hosting the Cepheids, in order to confirm membership of Cepheids in OCs and refine cluster's physical parameters (Majaess et al. 2013a,b, 2012, Turner et al. 2012).

One of the most interesting results of the studies of individual clusters was published by Davies et al. (2011) on GLIMPSE-C01 referred to in the literature as an old globular cluster traversing the Galactic disk. The authors obtained high-resolution near-infrared spectroscopy of over 50 stars in the cluster and found the average radial velocity is consistent with being part of the disk, and determined the cluster's dynamical mass to be $8 \times 10^{4} M_{\odot}$. From analysis of the cluster's $M / L$ ratio and location of the red clump, Davies et al. (2011) suggested the cluster's age to be 400-800 Myr and concluded that GLIMPSE-C01 is the most massive Galactic intermediate-age cluster discovered to date.

This way, the number of known Milky Way open clusters increased from about 1500 to almost 4000 during the last $10-15$ years. The main physical parameters were derived for most of them but quality of these determinations is insufficient to study the Galactic disk comprehensively. That is why a lot of new different surveys were started to measure a large range of open cluster's properties.

\section{Globular clusters in the Milky Way}

\section{Angela Bragaglia $\&$ Eugenio Carretta, Osservatorio di Bologna, Italy}

The last ten years have seen a renewed interest in Galactic globular clusters (GC), mainly because they have been demonstrated to be much more complex and intriguing than believed in the past. The main reason is that spectroscopic and, later on, photometric observations have driven a dramatic shift from considering GCs as the best approximation of simple stellar populations (see e.g. the review by Renzini \& Fusi Pecci 1988) to the simplest example of multiple stellar populations (e.g. the review by Gratton et al. 2004). We use here the words "populations" and "generations" as synonym, implying that in the same GC stars of (slightly) different age coexist. This is the generally accepted scenario, although there are still many problems in explaining the mechanism of GC formation and internal self-enrichment (in light elements for all GCs, in heavier elements only for a fraction). For recent reviews see for instance Gratton et al. (2012) for spectroscopic results, Piotto (2009) for photometry, and Charbonnel (2015) for theoretical challenges.

We present here selected highlights in Galactic GC work of the last decade, with a strong bias towards multiple populations and observations.

The abundance of light elements in GC stars shows large star-to-star scatter, at variance with what happens for the bulk of field halo stars; these variations are anti-correlated ( $\mathrm{C}$ and $\mathrm{N}, \mathrm{O}$ and $\mathrm{Na}$, and $\mathrm{Mg}$ and $\mathrm{Al}$ are depleted and enhanced, respectively). These so called "anomalies" in light elements had already been detected in many GCs, but generally only in giant stars. However, the presence of products of hot H-burning also in main sequence stars called for more massive stars as original polluters (see subsection on models) and we now speak of first-generation (FG) and second-generation (SG) stars in GCs. After the first pioneering works, light element (anti-)correlations were routinely found also among main sequence and unevolved stars, using also high-resolution spectra, see e.g. Carretta et al. (2005); Cohen et al. (2005); Kayser et al. (2006, 2008); Pancino et al. (2010); Smolinski et al. (2011); Lardo et al. (2012) for C, N, and Pasquini et al. (2005); Lind et al. (2009); Bragaglia et al. (2010); D'Orazi et al. (2010); Monaco et al. (2012); Dobrovolskas et al. (2014) for $\mathrm{Na}, \mathrm{O}, \mathrm{Mg}, \mathrm{Al}$, and Li. This decade saw a wealth of observations on large samples of stars and of clusters, also thanks to multi-object 
high-resolution spectrographs, like Hydra or FLAMES, so that a quantitative analysis of the light elements anti-correlation became possible. Large scale studies were conducted, see the long list of GCs and papers in Gratton et al. 2012, among which we have for instance the closest GCs, i.e., M 4, NGC 6752, NGC 6397, and M 22 (e.g. Marino et al. 2008; Carretta et al. 2009b,a; Yong et al. 2008; Korn et al. 2007; Marino et al. 2011b), very massive clusters like $\omega$ Cen (Marino et al. 2011a) and M 54 Carretta et al. (2010a), and low mass GCs like NGC6838 (Cordero et al. 2015). Recently, also high-resolution surveys produced results in this field (Mészáros et al. 2015; San Roman et al. 2015). All evolutionary phases were targeted. The red giant branch (RGB) was the favourite, but also the horizontal branch (HB) was observed and in some GCs also He was measured (see e.g. Villanova et al. 2009; Marino et al. 2014; Gratton et al. 2015), an important diagnostics of the multipopulation scenario. Helium has also been measured in RGB stars, but only in NGC 2808 (Pasquini et al. 2011) and $\omega$ Cen (Dupree \& Avrett 2013). More recently, the Asymptotic Giant Branch (AGB) observations showed that at least in some GCs the AGB stars do not show the same level of modification in light element abundances (Campbell et al. 2013; Charbonnel et al. 2013, but see also Johnson et al. 2015). Briefly, in all the examined MW GCs (and interestingly, also in GCs of the LMC (Johnson et al. 2006; Mucciarelli et al. 2009 and Fornax Letarte et al. 2006), a prominent $\mathrm{Na}-\mathrm{O}$ anti-correlation has been found. Possible, rare, exceptions are very low-mass GCs, like the two Sgr clusters Ter 7 and Pal 12 (e.g. Sbordone et al. 2005), and Rup106 (Villanova et al. 2013). The Na-O anti-correlation appears almost a defining properties of (massive) GCs and is not present among field stars or open clusters (Bragaglia et al. 2012, 2014; Cunha et al. 2015; MacLean et al. 2015, but see Geisler et al. 2012 for a different opinion on NGC6791). An even more extreme departure from the simple stellar population paradigma comes from the finding of GCs with dispersion in iron and heavy elements. After $\omega$ Cen, also M 22 was found to display an intrinsic metallicity spread by Da Costa et al. (2009); Marino et al. (2009) (recently challenged by Mucciarelli et al. 2015), correlated with a spread in neutron-capture elements. Among GCs displaying iron and n-capture element spreads are for instance M 54, NGC 1851, and M 22.

The impact of different abundances of light element ions the photometric properties of stars in GCs (especially in the filters containing molecular features of CNO elements, see Sbordone et al. e.g. 2011) is exploited to better understand the origin of GCs. Different abundances between FG and SG stars translate into spread and even split sequences along the whole CMD of GCs. These multiple sequences are observed with several photometric systems, including broad band (e.g. Marino et al. 2008; Milone et al. 2008; Han et al. 2009; Lardo et al. 2011), intermediate band (Yong et al. 2008; Carretta et al. 2011), and narrow band (Lee et al. 2009; Lim et al. 2015b). The recent exploitation of the UV Hubble Space telescope (HST) filters allows to reach also features of OH hydride (see the UV GC survey described in Piotto et al. 2015). HST observations reveal split sequences all the way from the main sequence (MS) up to the red giant branch (RGB) and horizontal brand (HB, see for instance the spectacular main sequence (MS) of NGC 2808, Piotto et al. 2007). The large photometric samples allow to study the radial distribution of stellar populations across the cluster area (e.g. Lardo et al. 2011; Milone et al. 2013; Kravtsov et al. 2011). Usually SG stars are found more centrally concentrated, as predicted by most scenarios of GC formation. Photometry permits to detect even discrete populations in a single GC, like NGC 2808 (Milone et al. 2015), confirmed by abundance analysis of individual stars (Carretta 2015). The coupling between precise spectroscopy and photometry unravels a variegated landscape for Galactic GCs, that come in different flavours. Most GCs are monometallic (concerning iron and heavier elements). A growing number of objects shows a spread in Fe (see above). They also share a few other common properties: a correlation 
between $\mathrm{Ca}$ and $\mathrm{Fe}$ (suggesting enrichment by type II SNe), and an enhancement of elements from slow neutron-capture process observed among stars of the more metalrich cluster component. This suggests that, like $\omega$ Cen and M 54, the remnant nuclei of former dwarf galaxy (e.g. Bekki \& Freeman 2003; Bellazzini et al. 2008), these GCs may be the final products of cluster formation in a dwarf galaxy environment (e.g. Bekki \& Yong 2012). Maybe related to the multiple populations in GCs, in the LMC and SMC many intermediate-age clusters with extended or even split main sequence turn-off's and red clumps have been found (e.g. Milone et al. 2009; Girardi et al. 2009; Correnti et al. 2015). This may be due to extended star formation (e.g. Goudfrooij et al. 2011) or to stellar rotation (e.g. Bastian \& de Mink 2009) or binarity (e.g. Yang et al. 2011). No light elements anti-correlation has yet been found in these clusters (Mucciarelli et al. 2014).

Observational evidence of multiple stellar populations calls for some FG stars to have polluted material from which the SG stars formed. The most commonly discussed FG polluters are intermediate mass asymptotic giant branch (AGB) stars (Ventura \& D'Antona 2008, e.g.) and fast rotating massive stars (FRMS Decressin et al. 2007, e.g.), but also interactive massive binaries have been proposed (de Mink et al. 2009), very massive stars (Denissenkov \& Hartwick 2014), or early disk accretion (Bastian et al. 2013). However, multiple populations also pose tight constraints that challenge (m)any model(s) of GC formation. The currently observed ratio of FG to SG stars (about 1/3 and 2/3, Carretta et al. 2010b; Bastian \& Lardo 2015) is not easily reconciled with the amount of yields provided by any candidate FG polluter (see for instance Bastian et al. 2015). This "mass budget" problem was often circumvented by scenarios assuming that GCs were initially from 10 up to 100 times more massive than present-day GCs (Bekki et al. 2007; Decressin et al. 2007; D'Ercole et al. 2008, 2010; Carretta et al. 2010b; Schaerer \& Charbonnel 2011). However, evidence is growing that in external dwarf galaxies harbouring old GCs (showing multiple populations) these objects cannot have been more than 3-4 times more massive at their formation epoch, since they already account for about $25 \%$ of the galaxy mass in metal-poor stars (Larsen et al. 2012, 2014; Tudorica et al. 2015). Another severe challenge is posed by the very same existence of the observed anti-correlations, which require a certain amount of pristine gas to be mixed with ejecta of polluters to reproduce the observations. In some scenario (e.g. with AGBs, D'Ercole et al. 2010) dilution is mandatory since AGBs produce a correlation between $\mathrm{Na}$ and $\mathrm{O}$ abundance. From where this gas with primordial composition came after the GCs were swept by the first type II Supernovae is an issue still open, widely discussed but with no completely satisfactory answer up to now. A recent scenario proposed by Trenti et al. 2015 shows a possible way out, with blobs of pristine gas nearby the outcome of a major mini-halo mini-halo merger at high redshift, which could be later accreted refurbishing the evolving proto-GC with fresh reservoir of diluting matter. This mechanism could also be an attractive way to explain the discreteness observed both in CMDs and in the anticorrelations (see e.g. Milone et al. 2013, 2015 and Carretta et al. 2012; Carretta 2015, for NGC 6752 and NGC 2808).

Clusters lose mass and stars during their evolution (see, e.g., Baumgardt et al. 2008). Stars probably lost by GCs have been searched for in halo samples using the peculiar SG chemistry. CN band strength was used by Martell \& Grebel (2010); Martell et al. (2011) on Segue SDSS spectra, while enhanced $\mathrm{Na}$ and/or depleted $\mathrm{O}$ abundances were considered by Carretta et al. (2010b); Ramírez et al. (2012). These papers provided a fraction of SG-like halo stars of about 1.5 to $3 \%$. Recently, using data from the public spectroscopic survey Gaia-ESO Lind et al. (2015) found a probable GC escapee. For a discussion on the contribution of GC stars to the halo, see for instance Gratton et al. (2012). With the advent of large photometric surveys such as SDSS, Pan-STARRS, DES, VST ATLAS, 
VVV, etc new GCs have been found, see e.g., Laevens et al. (2014); Belokurov et al. (2014), even if the objects found are sometimes classified as GCs or ultrafaint dwarfs depending on the study. The Galactic GC populations has increased in number, but the newly discovered clusters are all low-mass systems. Wide field imaging has been used systematically to look for extended and extra-tidal structures (e.g. Jordi \& Grebel 2010; Carballo-Bello et al. 2012) In a few cases, tidal tails or streams still connected to the originating GC have been found (e.g., NGC 288, Grillmair et al. 2013 or Pal 14, Sollima et al. 2011), but there are many more without a clearly associated progenitor cluster. Furthermore, streams, moving groups, common proper motions groups have been sometimes associated to now dissolved clusters, in some cases using chemical tagging. Examples of a positive and negative identification, respectively, are the Aquarius stream (consistent with being GC debris, Wylie-de Boer et al. 2012) and the Kapteyn group (not associated with $\omega$ Cen, Navarrete et al. 2015).

"Exotic" objects in GCs comprise for instance blue stragglers stars (BSS), low-mass $\mathrm{X}$-rays binaries, and millisecond pulsars (MSP), all very good tracers of the evolution of close binary systems in dense environments and of the dynamical history of the parent cluster. The study of BSS proceeded with the collection of large samples covering the entire extension of the GCs (e.g. Dalessandro et al. 2008; Leigh et al. 2011; Salinas et al. 2012), in the determination of their dynamical status (e.g. Ferraro et al. 2009a; Simunovic \& Puzia 2014; Ferraro et al. 2012) and chemical composition (e.g. Lovisi et al. 2012). For more detailed and recent highlights on BSS, see for instance the contributions in 'Ecology of Blue Straggler Stars', held in 2012. Ransom et al. (2014) found 21 new MSP in tbe massive GC Terzan 5, which shows the largest number of X-ray sources among GCs. This cluster is particularly interesting also because Ferraro et al. (2009b) found it hosts two stellar populations with different iron contents and ages and proposed it to be the remnant of one of the primordial building blocks that formed the bulge (and not a true GC). The different metallicity was later confirmed by Origlia et al. (2013); Massari et al. (2014).

\section{Extragalactic star clusters}

\section{Tom Richtler, Universidad de Concepción, Chile}

The following pages try to extract the results from 10 years of world wide intensive research using the extremely short style of a conference summary and applying a strong bias to observational work. Brodie \& Strader (2006) review "Globular clusters and Galaxy Formation", Harris (2010) "massive star clusters in galaxies", Portegies Zwart et al. (2010) "young massive clusters", Kruijssen (2014) concepts of GC formation.

New M31 GCs have been detected, many within the "Pan-Andromeda Archaeological Survey" (McConnachie et al. 2009) and the data base for M31 GCs has been significantly increased both in quantity and quality (Puzia et al. 2005; Kim et al. 2007; Lee et al. 2008; Fan et al. 2010; Huxor et al. 2014; di Tullio Zinn \& Zinn 2013, 2015). A updated analysis of the properties of the M31 GCS is given by Huxor et al. (2011). A flattening of the surface density profile at a radius of about $30 \mathrm{kpc}$ corresponds to a flattening in the stellar surface brightness profile, which might indicate an accretion of the outer halo. More direct evidence for accreted clusters is found in Mackey et al. (2010, 2013). Structural parameters are presented by Barmby et al. (2007); Peacock et al. (2010). 300 metallicities of old GCs have been derived by (Caldwell et al. 2011). The metallicity distribution is unimodal in contrast to that of the Milky Way and giant ellipticals. Detailed element abundances for GCs from integrated light have been derived by Colucci et al. (2009). 
Strader et al. (2011) combine kinematic data and structure parameters to derive $M / L$ ratios for $200 \mathrm{GCs}$ and confirmed previous findings that the $M / L_{V}$-values decline with increasing metallicity, contrary to what naively is expected from stellar models. Shallower mass functions of metal-rich clusters can explain this. $M / L_{V}$-values also increase with cluster mass, possibly as a consequence of mass segregation. This is disputed by Shanahan \& Gieles (2015). The kinematics of the outer halo GCs in M31 is studied by Veljanoski et al. (2014). Groups of GCs are related to the debris of stellar streams. The Fornax dwarf spheroidal shows an extremely large ratio of stars in GC to field stars of a similar low metallicity. This constrains the loss of first generation stars in GCs Larsen et al. (2012).

Nearby spirals with high star formation rates have been surveyed for clusters by Bastian et al. (2012) (M83), Larsen et al. (2011) used HST data to measure colour magnitude diagrams for resolved young massive clusters in nearby spiral galaxies. There is no gap visible between the H-burning main sequence stars and the He-burning supergiants like in canonical isochrones. Age spreads of a few Myrs are able to fill this gap, may be also interacting binaries.

The Initial Cluster Mass Function (ICMF) of young clusters in spiral galaxies has been investigated by Larsen (2009). A original Schechter function with a cut-off mass of $\approx 2 \times 10^{5} M_{\odot}$ describes the GC luminosity functions well, if the luminosity evolution is only secular. (Elmegreen 2006) provides insight into the similarity of cluster and stellar IMFs. Based on a sample of 37 nearby dwarf galaxies, Cook et al. (2012) study the relation between SFR and cluster formation. Galaxy mergers can host a plethora of massive clusters. The Antennae galaxies are prominent targets with age distribution of GCs studied by Fall et al. 2005, new spectroscopic data (Whitmore et al. 2005; Bastian et al. 2009), general demographic model and application to the antennae (Whitmore et al. 2007). The latter work demonstrates that the enhanced number of massive clusters in mergers is a sample size effect due to the high star formation rate. The Antennae are also a test ground for the evolution of clusters, in particular the processes of disruption (Fall et al. 2009; Renaud et al. 2008; Karl et al. 2011; Renaud \& Gieles 2013). Some young clusters in the Antennae have been observed with ALMA, but large reservoirs of molecular gas in the clusters have not been found (Cabrera-Ziri et al. 2015). Cluster formation and disruption in mergers through simulations have been investigated by Kruijssen et al. (2012). (Bastian et al. 2013) give structural parameters for 36 clusters in NGC 7572. Among them is W3, the most massive cluster known, whose profile extends out to 500 pc.

The nearest merger remnant (and giant elliptical) is NGC 5128 (CenA). Ages, metallicities are known for about 400 objects (Gómez et al. 2006; Rejkuba et al. 2007; Woodley et al. 2010a,b). One third of this sample show ages less than 8 Gyr. Taylor et al. (2010) find an increase of the M/L-values with the dynamical mass. The GCS has been searched over an area of $1.5 \mathrm{deg}^{2}$ (Harris et al. 2012). More than 1000 GCs are now known. NGC1316 (Fornax A) is after CenA the closest merger remnant. Its GCS host clusters of a wide age-range down to $0.5 \mathrm{Gyr}$ (Richtler et al. 2014). Among them are objects as massive as $1.6 \times 10^{7} M_{\odot}$ (Bastian et al. 2006). There is an isolated young star cluster complex which demonstrates GC formation outside periods of high star formation rate (Richtler et al. 2012).

The nearby galaxy clusters Virgo and Fornax have been intensively surveyed with HST/ACS which produced a bulk of papers. From the Virgo survey: Peng et al. (2008) study formation efficiencies of GCs. The GC mass fraction is lowest at intermediate host luminosity, the specific frequency is dominated by blue clusters. Dwarf galaxies near to M87 seem to be tidally stripped of their GCs. Villegas et al. (2010) on the GC luminosity 
function and distance determination: the dispersion of the GCLF correlated tightly with the host's absolute magnitude. Mieske et al. (2006) on colour-magnitude relations in GCSs, Liu et al. (2011) on colour gradients within GCSs. Georgiev et al. (2010) find that the total mass in GCs scales with the halo mass of the host galaxy.

A new catalogue by Harris et al. (2013) updates our current knowledge of GCS properties and relations with host galaxy properties. The number of GCs obeys a fundamental plane-like relation $N_{G C} \sim\left(R_{e} \sigma_{e}\right)^{1.3}$ for galaxies of all luminosities. On the other hand the ratio mass in GCs/halo mass is essentially constant (Hudson et al. 2014), the GC number also scales with halo dark mass and Harris et al. (2015) even find a strict proportionality between the number of blue clusters and the halo mass. Harris et al. (2014b) compare the GC luminosity functions of seven brightest cluster galaxies and find identical shapes.

Dwarf galaxies show a tendency of increasing specific frequency with decreasing luminosity (Miller \& Lotz 2007). How the GCSs of dwarf galaxies are affected by galaxy harassment in clusters, is studied by Smith et al. (2013). The critical parameter is the dark matter fraction that remains after interaction processes. Brüns et al. (2011) explain both compact and extended clusters by dynamical evolution in star cluster complexes. Massive young GCs are also seen in star forming dwarf galaxies (Adamo et al. 2011) which in the context of hierarchical clustering may have been important for the assembly of GCSs.

The richest GCs of nearby galaxies are found in the central galaxies of the Virgo (Harris 2009) and Fornax galaxy clusters. As population and dynamical tracers GCs have a high significance for investigating the dark matter content and distribution as well as the population structure and formation history of M87 and NGC 1399. Regarding NGC 1399, Schuberth et al. (2010) presented about $700 \mathrm{GC}$ radial velocities within $80 \mathrm{kpc}$ of galactocentric radius. Blue and red clusters show distinct kinematical properties with a sharp transition. The red clusters resemble the galaxy light, while blue clusters behave more erratic and are probably accreted. The best fitting dark halo agrees reasonably well with that from X-ray studies, but a substructure within the dark halo that has been suspected in earlier X-ray studies, has not been confirmed.

M87 also received particular attention. Strader et al. (2011) provide a wealth of kinematical data for over 700 GCs that Agnello et al. (2014) use for a dynamical analysis. They found a dark matter fraction of 0.95 within a radius of $135 \mathrm{kpc}$ and an inner dark matter profile that is steeper than predicted by cosmological simulations. Such a big sample also permits to analyse the orbital properties of GCs. Remarkably, the anisotropy seems to be mainly tangential which supports the idea that many objects on radial orbits have been dissolved. Near M87 has been found the object with the highest negative radial velocity detected so far, a GC with $-1025 \mathrm{~km} / \mathrm{s}$ (Caldwell et al. 2014). Its dynamical history is mysterious.

A significant increase of the database regarding metallicities (Usher et al. 2012) and kinematics of GCs in early-type galaxies (Pota et al. 2013a) was achieved by the still ongoing "The SAGES Legacy Unifying Globulars and GalaxieS Survey (SLUGGS)" (Brodie et al. 2014). Until now, almost 1000 metallicities and 2500 velocities of GCs in a dozen early-type galaxies have been published. Highlighting a few galaxies: The disputed metallicity bimodality of GCs in NGC 3115 has been confirmed by Brodie et al. (2012) through CaII triplet strengths. Breaking the degeneracy in dynamical models between potential and orbital anisotropy, (Napolitano et al. 2014) find the dark halo of NGC 5846 to be consistent with cosmological simulations, that its stellar IMF is Salpeter-like, and that the GC orbits are isotropic in the central parts and slightly radial at larger distances (Napolitano et al. 2014). More than 400 GCs with photometry and velocities build the database for M60 (Pota et al. 2015). 
The correlation between central supermassive black hole masses and properties of GCSs have been discussed in several papers (Burkert \& Tremaine 2010; Sadoun \& Colin 2012; Pota et al. 2013b; Harris et al. 2014a). The latter two contributions could investigate larger galaxy samples and find the correlations weaker than described before. Massive nuclear GCs host supermassive black holes. Graham \& Spitler (2009) provides a relation between the masses of a nuclear star cluster, the bulge and the black hole. Antonini et al. (2015) show how erosion by binary BHs may change the mass of a nuclear star cluster. Georgiev \& Böker (2014) provides a catalogue of 228 nuclear star clusters in nearby spirals.

The discussion regarding the characteristic parameters and the nature of UCDs is continuing. Brodie et al. (2011) find, on the basis of a survey in M87, that the colourmagnitude relation of UCDs is offset from that of GCs. They conclude that the majority of UCDs are stripped nuclei of former dwarf galaxies. Norris \& Kannappan (2011) point to the dual nature of UCDs characterised by mass. Above $7 \times 10^{7} M_{\odot}$, UCDs are predominantly stripped nuclei, while at lower masses, many "normal" GCs may be mixed in. An interesting finding were the enhanced M/L-values of some UCDs in the Virgo cluster which could not be explained by stellar population models, perhaps indicative of dark matter (Haşegan et al. 2005). Later work on UCDs in the Fornax cluster did not confirm this (Hilker et al. 2007). In particular, the brightest and resolved UCD in the Fornax cluster shows population properties consistent with existing models Frank et al. (2011), but the discussion is going on Mieske et al. (2013). The study of a large number of UCDs around M87 suggests that UCDs are distinct from GCs by their sizes rather than by their masses. The UCDs in M87 show both radial distributions and orbital properties different from those of GCs (Zhang et al. 2015). Brüns et al. (2011) explain both compact and extended clusters by dynamical evolution in star cluster complexes. Convincing evidence that some UCDs evolve from bigger parent galaxies, is the detection of a supermassive black hole in a UCD in M60 that has $15 \%$ of the mass of its host system Seth et al. (2014).

Cosmological formation of GCs has not yet been identified, but GCs populating a galaxy cluster rather than an individual galaxy, have been found by Peng et al. (2011) in the Coma cluster, West et al. (2011) in Abell 1189, and by Alamo-Martínez et al. (2013) in Abell 1689. For the latter cluster, the authors quote a number of $160000 \mathrm{GC}$ within $400 \mathrm{kpc}$. The other extreme appears as "the most isolated globular clusters in the Local Universe" in the vicinities of M81 and M82 Jang et al. (2012). The Local Group does not seem to host very isolated GCs (di Tullio Zinn \& Zinn 2013).

\section{References}

Adamo, A., Östlin, G., Zackrisson, E., \& Hayes, M. 2011, MNRAS, 414, 1793

Agnello, A., Evans, N. W., Romanowsky, A. J., \& Brodie, J. P. 2014, MNRAS, 442, 3299

Alamo-Martínez, K. A., Blakeslee, J. P., Jee, M. J., et al. 2013, ApJ, 775, 20

Anderson, L. D., Bania, T. M., Balser, D. S., \& Rood, R. T. 2011, ApJS, 194, 32

Anderson, R. I., Eyer, L., \& Mowlavi, N. 2013, MNRAS, 434, 2238

Anguiano, B., Freeman, K., Bland-Hawthorn, J., et al. 2014, in IAU Symposium, Vol. 298, IAU

Symposium, ed. S. Feltzing, G. Zhao, N. A. Walton, \& P. Whitelock, 322-325

Antonini, F., Barausse, E., \& Silk, J. 2015, ApJ, 806, L8

Ascenso, J., Alves, J., Vicente, S., \& Lago, M. T. V. T. 2007, A\&A, 476, 199

Bally, J., Anderson, L. D., Battersby, C., et al. 2010, A\&A, 518, L90

Barbá, R. H., Roman-Lopes, A., Nilo Castellón, J. L., et al. 2015, A\&A, 581, A120

Barmby, P., McLaughlin, D. E., Harris, W. E., Harris, G. L. H., \& Forbes, D. A. 2007, AJ, 133, 2764 
Bastian, N., Adamo, A., Gieles, M., et al. 2012, MNRAS, 419, 2606

Bastian, N., Cabrera-Ziri, I., \& Salaris, M. 2015, MNRAS, 449, 3333

Bastian, N. \& de Mink, S. E. 2009, MNRAS, 398, L11

Bastian, N. \& Lardo, C. 2015, MNRAS, 453, 357

Bastian, N., Saglia, R. P., Goudfrooij, P., et al. 2006, A\&AA, 448, 881

Bastian, N., Schweizer, F., Goudfrooij, P., Larsen, S. S., \& Kissler-Patig, M. 2013, MNRAS, 431, 1252

Bastian, N., Trancho, G., Konstantopoulos, I. S., \& Miller, B. W. 2009, ApJ, 701, 607

Baumgardt, H., Kroupa, P., \& Parmentier, G. 2008, MNRAS, 384, 1231

Bekki, K., Campbell, S. W., Lattanzio, J. C., \& Norris, J. E. 2007, MNRAS, 377, 335

Bekki, K. \& Freeman, K. C. 2003, MNRAS, 346, L11

Bekki, K. \& Yong, D. 2012, MNRAS, 419, 2063

Beletsky, Y., Carraro, G., \& Ivanov, V. D. 2009, A\&A A, 508, 1279

Bellazzini, M., Ibata, R. A., Chapman, S. C., et al. 2008, AJ, 136, 1147

Belokurov, V., Irwin, M. J., Koposov, S. E., et al. 2014, MNRAS, 441, 2124

Bica, E., Dutra, C. M., Soares, J., \& Barbuy, B. 2003, A\&A, 404, 223

Bik, A., Henning, T., Stolte, A., et al. 2012, ApJ, 744, 87

Borissova, J., Bonatto, C., Kurtev, R., et al. 2011, A\&A, 532, A131

Borissova, J., Chené, A.-N., Ramírez Alegría, S., et al. 2014, A\&SA, 569, A24

Borissova, J., Clarke, J. R. A., Bonatto, C., et al. 2012, in Astronomical Society of the Pacific Conference Series, Vol. 465, Proceedings of a Scientific Meeting in Honor of Anthony F. J.

Moffat, ed. L. Drissen, C. Robert, N. St-Louis, \& A. F. J. Moffat, 504

Bragaglia, A., Carretta, E., Gratton, R. G., et al. 2010, ApJ, 720, L41

Bragaglia, A., Gratton, R. G., Carretta, E., et al. 2012, A\&3A, 548, A122

Bragaglia, A., Sneden, C., Carretta, E., et al. 2014, ApJ, 796, 68

Bragaglia, A. \& Tosi, M. 2006, AJ, 131, 1544

Brodie, J. P., Romanowsky, A. J., Strader, J., \& Forbes, D. A. 2011, AJ, 142, 199

Brodie, J. P., Romanowsky, A. J., Strader, J., et al. 2014, ApJ, 796, 52

Brodie, J. P. \& Strader, J. 2006, ARA\&A, 44, 193

Brodie, J. P., Usher, C., Conroy, C., et al. 2012, ApJ, 759, L33

Brüns, R. C., Kroupa, P., Fellhauer, M., Metz, M., \& Assmann, P. 2011, A\&A, 529, A138

Bukowiecki, Ł., Maciejewski, G., Konorski, P., \& Strobel, A. 2011, Acta Astron., 61, 231

Burkert, A. \& Tremaine, S. 2010, ApJ, 720, 516

Cabrera-Ziri, I., Bastian, N., Longmore, S. N., et al. 2015, MNRAS, 448, 2224

Caetano, T. C., Dias, W. S., Lépine, J. R. D., et al. 2015, New A, 38, 31

Caldwell, N., Schiavon, R., Morrison, H., Rose, J. A., \& Harding, P. 2011, AJ, 141, 61

Caldwell, N., Strader, J., Romanowsky, A. J., et al. 2014, ApJ, 787, L11

Camargo, D., Bica, E., Bonatto, C., \& Salerno, G. 2015, MNRAS, 448, 1930

Campbell, S. W., D'Orazi, V., Yong, D., et al. 2013, Nature, 498, 198

Carballo-Bello, J. A., Gieles, M., Sollima, A., et al. 2012, MNRAS, 419, 14

Carraro, G., Turner, D., Majaess, D., \& Baume, G. 2013, A\&̈A, 555, A50

Carretta, E. 2015, ApJ, 810, 148

Carretta, E., Bragaglia, A., Gratton, R., D’Orazi, V., \& Lucatello, S. 2011, A\&̊A, 535, A121

Carretta, E., Bragaglia, A., Gratton, R., \& Lucatello, S. 2009a, A\&\&A, 505, 139

Carretta, E., Bragaglia, A., Gratton, R. G., et al. 2010a, A\&BA, 520, A95

Carretta, E., Bragaglia, A., Gratton, R. G., et al. 2009b, A\&SA, 505, 117

Carretta, E., Bragaglia, A., Gratton, R. G., Lucatello, S., \& D'Orazi, V. 2012, ApJ, 750, L14

Carretta, E., Bragaglia, A., Gratton, R. G., et al. 2010b, A $\& A$, 516, A55

Carretta, E., Gratton, R. G., Lucatello, S., Bragaglia, A., \& Bonifacio, P. 2005, A 8 A, 433, 597

Casamiquela, L., Carrera, R., Jordi, C., \& Balaguer-Núñez, L. 2014, in EAS Publications Series, Vol. 67, EAS Publications Series, 361-361

Charbonnel, C. 2015, IAU General Assembly, 22, 87893

Charbonnel, C., Chantereau, W., Decressin, T., Meynet, G., \& Schaerer, D. 2013, A\&\&A, 557, L17 
Chen, X., de Grijs, R., \& Deng, L. 2015, MNRAS, 446, 1268

Chené, A.-N., Borissova, J., Bonatto, C., et al. 2013, A\&GA, 549, A98

Churchwell, E., Babler, B. L., Meade, M. R., et al. 2009, PASP, 121, 213

Churchwell, E., Povich, M. S., Allen, D., et al. 2006, ApJ, 649, 759

Clark, J. S., Negueruela, I., Crowther, P. A., \& Goodwin, S. P. 2005, A\& A, 434, 949

Clark, J. S., Ritchie, B. W., Najarro, F., Langer, N., \& Negueruela, I. 2014, A\&A, 565, A90

Clarkson, W. I., Ghez, A. M., Morris, M. R., et al. 2012, ApJ, 751, 132

Cohen, J. G., Briley, M. M., \& Stetson, P. B. 2005, AJ, 130, 1177

Colucci, J. E., Bernstein, R. A., Cameron, S., McWilliam, A., \& Cohen, J. G. 2009, ApJ, 704, 385

Conrad, C., Scholz, R.-D., Kharchenko, N. V., et al. 2014, A\& A, 562, A54

Cook, D. O., Seth, A. C., Dale, D. A., et al. 2012, ApJ, 751, 100

Cordero, M. J., Pilachowski, C. A., Johnson, C. I., \& Vesperini, E. 2015, ApJ, 800, 3

Correnti, M., Goudfrooij, P., Puzia, T. H., \& de Mink, S. E. 2015, MNRAS, 450, 3054

Costa, E., Moitinho, A., Radiszc, M., et al. 2015, A\&A, 580, A4

Cottaar, M., Covey, K. R., Meyer, M. R., et al. 2014, ApJ, 794, 125

Covey, K. R., Cottaar, M., Foster, J. B., et al. 2015, in American Astronomical Society Meeting Abstracts, Vol. 225, Bulletin of American Astronomical Society, 302.03

Cunha, K., Smith, V. V., Johnson, J. A., et al. 2015, ApJ, 798, L41

Da Costa, G. S., Held, E. V., Saviane, I., \& Gullieuszik, M. 2009, ApJ, 705, 1481

Dalessandro, E., Lanzoni, B., Ferraro, F. R., et al. 2008, ApJ, 681, 311

Davies, B., Bastian, N., Gieles, M., et al. 2011, MNRAS, 411, 1386

Davies, B., de La Fuente, D., Najarro, F., et al. 2012, MNRAS, 419, 1860

Davies, B., Figer, D. F., Kudritzki, R.-P., et al. 2007, ApJ, 671, 781

de Mink, S. E., Pols, O. R., Langer, N., \& Izzard, R. G. 2009, A\&A, 507, L1

Decressin, T., Meynet, G., Charbonnel, C., Prantzos, N., \& Ekström, S. 2007, A\& A, 464, 1029

Deharveng, L., Schuller, F., Anderson, L. D., et al. 2010, A\&A, 523, A6

Denissenkov, P. A. \& Hartwick, F. D. A. 2014, MNRAS, 437, L21

D'Ercole, A., D'Antona, F., Ventura, P., Vesperini, E., \& McMillan, S. L. W. 2010, MNRAS, 407,854

D'Ercole, A., Vesperini, E., D'Antona, F., McMillan, S. L. W., \& Recchi, S. 2008, MNRAS, 391, 825

di Tullio Zinn, G. \& Zinn, R. 2013, AJ, 145, 50

di Tullio Zinn, G. \& Zinn, R. 2015, AJ, 149, 139

Dias, W. S., Monteiro, H., Caetano, T. C., et al. 2014, A\& A, 564, A79

Dobrovolskas, V., Kučinskas, A., Bonifacio, P., et al. 2014, A $₫ A, 565$, A121

D'Orazi, V., Lucatello, S., Gratton, R., et al. 2010, ApJ, 713, L1

Drake, A. J. 2005, A\&A A, 435, 545

Dupree, A. K. \& Avrett, E. H. 2013, ApJ, 773, L28

Dutra, C. M., Bica, E., Soares, J., \& Barbuy, B. 2003, A\&A, 400, 533

Elmegreen, B. G. 2006, ApJ, 648, 572

Evans, C. J., Smartt, S. J., Lee, J.-K., et al. 2005, A\&A, 437, 467

Evans, C. J., Taylor, W. D., Hénault-Brunet, V., et al. 2011, A\&AA, 530, A108

Fall, S. M., Chandar, R., \& Whitmore, B. C. 2005, ApJ, 631, L133

Fall, S. M., Chandar, R., \& Whitmore, B. C. 2009, ApJ, 704, 453

Fan, Z., de Grijs, R., \& Zhou, X. 2010, ApJ, 725, 200

Faria, M. A. F., Monteiro, H., Dias, W. S., \& Lépine, J. R. D. 2014, in Revista Mexicana de Astronomia y Astrofisica Conference Series, Vol. 44, Revista Mexicana de Astronomia y Astrofisica Conference Series, 175-176

Feigelson, E. D., Townsley, L. K., Broos, P. S., et al. 2013, ApJS, 209, 26

Ferraro, F. R., Beccari, G., Dalessandro, E., et al. 2009a, Nature, 462, 1028

Ferraro, F. R., Dalessandro, E., Mucciarelli, A., et al. 2009b, Nature, 462, 483

Ferraro, F. R., Lanzoni, B., Dalessandro, E., et al. 2012, Nature, 492, 393

Figer, D. F., MacKenty, J. W., Robberto, M., et al. 2006, ApJ, 643, 1166 
Frank, M. J., Hilker, M., Mieske, S., et al. 2011, MNRAS, 414, L70

Frinchaboy, P. M., Thompson, B. A., O'Connell, J., et al. 2015, in American Astronomical Society Meeting Abstracts, Vol. 225, American Astronomical Society Meeting Abstracts, 340.03

Froebrich, D., Scholz, A., \& Raftery, C. L. 2007, MNRAS, 374, 399

Geisler, D., Villanova, S., Carraro, G., et al. 2012, ApJ, 756, L40

Gennaro, M., Brandner, W., Stolte, A., \& Henning, T. 2011, MNRAS, 412, 2469

Georgiev, I. Y. \& Böker, T. 2014, MNRAS, 441, 3570

Georgiev, I. Y., Puzia, T. H., Goudfrooij, P., \& Hilker, M. 2010, MNRAS, 406, 1967

Gilmore, G., Randich, S., Asplund, M., et al. 2012, The Messenger, 147, 25

Girardi, L., Rubele, S., \& Kerber, L. 2009, MNRAS, 394, L74

Glushkova, E. V., Koposov, S. E., Zolotukhin, I. Y., et al. 2010, Astronomy Letters, 36, 75

Gómez, M., Geisler, D., Harris, W. E., et al. 2006, A\&A A, 447, 877

Goudfrooij, P., Puzia, T. H., Chandar, R., \& Kozhurina-Platais, V. 2011, ApJ, 737, 4

Graham, A. W. \& Spitler, L. R. 2009, MNRAS, 397, 2148

Gratton, R., Sneden, C., \& Carretta, E. 2004, ARA $\mathcal{E A}$, 42, 385

Gratton, R. G., Carretta, E., \& Bragaglia, A. 2012, A\&A Rev., 20, 50

Gratton, R. G., Lucatello, S., Sollima, A., et al. 2015, A\&SA, 573, A92

Grillmair, C. J., Cutri, R., Masci, F. J., et al. 2013, ApJ, 769, L23

Haşegan, M., Jordán, A., Côté, P., et al. 2005, ApJ, 627, 203

Han, S.-I., Lee, Y.-W., Joo, S.-J., et al. 2009, ApJ, 707, L190

Harayama, Y., Eisenhauer, F., \& Martins, F. 2008, ApJ, 675, 1319

Harris, G. L. H., Gómez, M., Harris, W. E., et al. 2012, AJ, 143, 84

Harris, G. L. H., Poole, G. B., \& Harris, W. E. 2014a, MNRAS, 438, 2117

Harris, W. E. 2009, ApJ, 703, 939

Harris, W. E. 2010, Philosophical Transactions of the Royal Society of London Series A, 368, 889

Harris, W. E., Harris, G. L., \& Hudson, M. J. 2015, ApJ, 806, 36

Harris, W. E., Harris, G. L. H., \& Alessi, M. 2013, ApJ, 772, 82

Harris, W. E., Morningstar, W., Gnedin, O. Y., et al. 2014b, ApJ, 797, 128

Heiter, U., Soubiran, C., Netopil, M., \& Paunzen, E. 2014, A\&3A, 561, A93

Hilker, M., Baumgardt, H., Infante, L., et al. 2007, A\&SA, 463, 119

Hindson, L., Thompson, M. A., Urquhart, J. S., et al. 2013, MNRAS, 435, 2003

Homeier, N. L. \& Alves, J. 2005, A\&A, 430, 481

Hudson, M. J., Harris, G. L., \& Harris, W. E. 2014, ApJ, 787, L5

Huxor, A. P., Ferguson, A. M. N., Tanvir, N. R., et al. 2011, MNRAS, 414, 770

Huxor, A. P., Mackey, A. D., Ferguson, A. M. N., et al. 2014, MNRAS, 442, 2165

Ivanov, V. D., Borissova, J., Bresolin, F., \& Pessev, P. 2005, A\&A, 435, 107

Ivanov, V. D., Borissova, J., Pessev, P., Ivanov, G. R., \& Kurtev, R. 2002, A\& $A$, 394, L1

Jang, I. S., Lim, S., Park, H. S., \& Lee, M. G. 2012, ApJ, 751, L19

Johnson, C. I., McDonald, I., Pilachowski, C. A., et al. 2015, AJ, 149, 71

Johnson, J. A., Ivans, I. I., \& Stetson, P. B. 2006, ApJ, 640, 801

Jordi, K. \& Grebel, E. K. 2010, $A \& A$, 522, A71

Karl, S. J., Fall, S. M., \& Naab, T. 2011, ApJ, 734, 11

Kayser, A., Hilker, M., Grebel, E. K., \& Willemsen, P. G. 2008, A\&AA, 486, 437

Kayser, A., Hilker, M., Richtler, T., \& Willemsen, P. G. 2006, A $\mathscr{E} A$, 458, 777

Kharchenko, N. V., Piskunov, A. E., Schilbach, E., Röser, S., \& Scholz, R.-D. 2013, A\& A, 558, A53

Kim, S. C., Lee, M. G., Geisler, D., et al. 2007, AJ, 134, 706

Kinemuchi, K., Sarajedini, A., Geisler, D., et al. 2010, in Bulletin of the American Astronomical Society, Vol. 42, American Astronomical Society Meeting Abstracts, 308.02

Koposov, S. E., Glushkova, E. V., \& Zolotukhin, I. Y. 2008, A\&A, 486, 771

Korn, A. J., Grundahl, F., Richard, O., et al. 2007, ApJ, 671, 402

Kravtsov, V., Alcaíno, G., Marconi, G., \& Alvarado, F. 2011, A\& A, 527, L9 
Kronberger, M., Teutsch, P., Alessi, B., et al. 2006, A\&A A, 447, 921

Kruijssen, J. M. D. 2014, Classical and Quantum Gravity, 31, 244006

Kruijssen, J. M. D., Pelupessy, F. I., Lamers, H. J. G. L. M., et al. 2012, MNRAS, 421, 1927

Kudryavtseva, N., Brandner, W., Gennaro, M., et al. 2012, ApJ, 750, L44

Kuhn, M. A., Feigelson, E. D., Getman, K. V., et al. 2014, ApJ, 787, 107

Laevens, B. P. M., Martin, N. F., Sesar, B., et al. 2014, ApJ, 786, L3

Lardo, C., Bellazzini, M., Pancino, E., et al. 2011, A\&AA, 525, A114

Lardo, C., Milone, A. P., Marino, A. F., et al. 2012, A\& $A$, 541, A141

Larsen, S. S. 2009, A\&\&A, 494, 539

Larsen, S. S., Brodie, J. P., Forbes, D. A., \& Strader, J. 2014, A\&AA, 565, A98

Larsen, S. S., de Mink, S. E., Eldridge, J. J., et al. 2011, A\&A, 532, A147

Larsen, S. S., Strader, J., \& Brodie, J. P. 2012, A\&A, 544, L14

Lee, J.-W., Kang, Y.-W., Lee, J., \& Lee, Y.-W. 2009, Nature, 462, 480

Lee, M. G., Hwang, H. S., Kim, S. C., et al. 2008, ApJ, 674, 886

Leigh, N., Sills, A., \& Knigge, C. 2011, MNRAS, 415, 3771

Letarte, B., Hill, V., Jablonka, P., et al. 2006, A\& $A$, 453, 547

Lim, B., Sung, H., Hur, H., \& Park, B.-G. 2015a, IAU General Assembly, 22, 46322

Lim, D., Han, S.-I., Lee, Y.-W., et al. 2015b, ApJS, 216, 19

Lind, K., Koposov, S. E., Battistini, C., et al. 2015, A\&A, 575, L12

Lind, K., Primas, F., Charbonnel, C., Grundahl, F., \& Asplund, M. 2009, A\&A, 503, 545

Liu, C., Peng, E. W., Jordán, A., et al. 2011, ApJ, 728, 116

Lovisi, L., Mucciarelli, A., Lanzoni, B., et al. 2012, ApJ, 754, 91

Mackey, A. D., Huxor, A. P., Ferguson, A. M. N., et al. 2010, ApJ, 717, L11

Mackey, A. D., Huxor, A. P., Ferguson, A. M. N., et al. 2013, MNRAS, 429, 281

MacLean, B. T., De Silva, G. M., \& Lattanzio, J. 2015, MNRAS, 446, 3556

Majaess, D., Carraro, G., Moni Bidin, C., et al. 2013a, A\&A, 560, A22

Majaess, D., Sturch, L., Moni Bidin, C., et al. 2013b, Ap\&SSS, 347, 61

Majaess, D., Turner, D. G., Gallo, L., et al. 2012, ApJ, 753, 144

Marco, A. \& Negueruela, I. 2011, A\&AA, 534, A114

Marco, A., Negueruela, I., González-Fernández, C., et al. 2014, A\&AA, 567, A73

Marino, A. F., Milone, A. P., Piotto, G., et al. 2009, A\&A, 505, 1099

Marino, A. F., Milone, A. P., Piotto, G., et al. 2011a, ApJ, 731, 64

Marino, A. F., Milone, A. P., Przybilla, N., et al. 2014, MNRAS, 437, 1609

Marino, A. F., Sneden, C., Kraft, R. P., et al. 2011b, A\&A, 532, A8

Marino, A. F., Villanova, S., Piotto, G., et al. 2008, A\&A A, 490, 625

Martell, S. L. \& Grebel, E. K. 2010, A\&AA, 519, A14

Martell, S. L., Smolinski, J. P., Beers, T. C., \& Grebel, E. K. 2011, A\&ऽA, 534, A136

Massari, D., Mucciarelli, A., Ferraro, F. R., et al. 2014, ApJ, 795, 22

Mathieu, R. D. \& WOCS Collaboration. 2013, in American Astronomical Society Meeting Abstracts, Vol. 222, American Astronomical Society Meeting Abstracts, 214.03

McConnachie, A. W., Irwin, M. J., Ibata, R. A., et al. 2009, Nature, 461, 66

Mercer, E. P., Clemens, D. P., Meade, M. R., et al. 2005, ApJ, 635, 560

Messineo, M., Clark, J. S., Figer, D. F., et al. 2015, ApJ, 805, 110

Messineo, M., Davies, B., Ivanov, V. D., et al. 2009, ApJ, 697, 701

Messineo, M., Figer, D. F., Davies, B., et al. 2010, ApJ, 708, 1241

Mészáros, S., Martell, S. L., Shetrone, M., et al. 2015, AJ, 149, 153

Mieske, S., Frank, M. J., Baumgardt, H., et al. 2013, A\&SA, 558, A14

Mieske, S., Jordán, A., Côté, P., et al. 2006, ApJ, 653, 193

Miller, B. W. \& Lotz, J. M. 2007, ApJ, 670, 1074

Milone, A. P., Bedin, L. R., Piotto, G., \& Anderson, J. 2009, A\&A, 497, 755

Milone, A. P., Bedin, L. R., Piotto, G., et al. 2008, ApJ, 673, 241

Milone, A. P., Marino, A. F., Piotto, G., et al. 2013, ApJ, 767, 120

Milone, A. P., Marino, A. F., Piotto, G., et al. 2015, ApJ, 808, 51

Monaco, L., Villanova, S., Bonifacio, P., et al. 2012, A\&\&A, 539, A157 
Morales, E. F. E., Wyrowski, F., Schuller, F., \& Menten, K. M. 2013, A\& $A$, 560, A76

Motte, F., Nguyên Luong, Q., Schneider, N., et al. 2014, A\&AA, 571, A32

Mottram, J. C., Hoare, M. G., Davies, B., et al. 2011, ApJ, 730, L33

Mucciarelli, A., Dalessandro, E., Ferraro, F. R., Origlia, L., \& Lanzoni, B. 2014, ApJ, 793, L6

Mucciarelli, A., Lapenna, E., Massari, D., et al. 2015, ApJ, 809, 128

Mucciarelli, A., Origlia, L., Ferraro, F. R., \& Pancino, E. 2009, ApJ, 695, L134

Napolitano, N. R., Pota, V., Romanowsky, A. J., et al. 2014, MNRAS, 439, 659

Navarrete, C., Chanamé, J., Ramírez, I., et al. 2015, ApJ, 808, 103

Negueruela, I., Clark, J. S., \& Ritchie, B. W. 2010, A\&A, 516, A78

Negueruela, I., González-Fernández, C., Marco, A., \& Clark, J. S. 2011, A\&̋A, 528, A59

Negueruela, I., Marco, A., González-Fernández, C., et al. 2012, A\&A, 547, A15

Netopil, M., Paunzen, E., \& Carraro, G. 2015, A\& A, 582, A19

Nguyen Luong, Q., Motte, F., Schuller, F., et al. 2011, A\&SA, 529, A41

Nishiyama, S., Tamura, M., Hatano, H., et al. 2009, ApJ, 696, 1407

Norris, M. A. \& Kannappan, S. J. 2011, MNRAS, 414, 739

Origlia, L., Massari, D., Rich, R. M., et al. 2013, ApJ, 779, L5

Pancino, E., Rejkuba, M., Zoccali, M., \& Carrera, R. 2010, A\& A, 524, A44

Panwar, N., Chen, W. P., Pandey, A. K., et al. 2014, MNRAS, 443, 1614

Pasquini, L., Bonifacio, P., Molaro, P., et al. 2005, A\&A, 441, 549

Pasquini, L., Mauas, P., Käufl, H. U., \& Cacciari, C. 2011, A\&A, 531, A35

Paunzen, E., Heiter, U., Netopil, M., \& Soubiran, C. 2010, A\&SA, 517, A32

Peacock, M. B., Maccarone, T. J., Knigge, C., et al. 2010, MNRAS, 402, 803

Peng, E. W., Ferguson, H. C., Goudfrooij, P., et al. 2011, ApJ, 730, 23

Peng, E. W., Jordán, A., Côté, P., et al. 2008, ApJ, 681, 197

Peri, C. S., Benaglia, P., Brookes, D. P., Stevens, I. R., \& Isequilla, N. L. 2012, AשA, 538, A108

Perren, G. I., Vázquez, R. A., \& Piatti, A. E. 2015, A\&SA, 576, A6

Pinheiro, M. C., Abraham, Z., Copetti, M. V. F., et al. 2012, MNRAS, 423, 2425

Piotto, G. 2009, in IAU Symposium, Vol. 258, IAU Symposium, ed. E. E. Mamajek, D. R. Soderblom, \& R. F. G. Wyse, 233-244

Piotto, G., Bedin, L. R., Anderson, J., et al. 2007, ApJ, 661, L53

Piotto, G., Milone, A. P., Bedin, L. R., et al. 2015, AJ, 149, 91

Pojmanski, G., Pilecki, B., \& Szczygiel, D. 2005, Acta Astron., 55, 275

Popescu, B. \& Hanson, M. M. 2010, in Bulletin of the American Astronomical Society, Vol. 42, American Astronomical Society Meeting Abstracts, 455.26

Popescu, B., Hanson, M. M., Borissova, J., et al. 2014, in Revista Mexicana de Astronomia y Astrofisica Conference Series, Vol. 44, Revista Mexicana de Astronomia y Astrofisica Conference Series, 163-163

Portegies Zwart, S. F., McMillan, S. L. W., \& Gieles, M. 2010, ARAESA, 48, 431

Pota, V., Brodie, J. P., Bridges, T., et al. 2015, MNRAS, 450, 1962

Pota, V., Forbes, D. A., Romanowsky, A. J., et al. 2013a, MNRAS, 428, 389

Pota, V., Graham, A. W., Forbes, D. A., et al. 2013b, MNRAS, 433, 235

Preibisch, T., Ratzka, T., Kuderna, B., et al. 2011, A\&SA, 530, A34

Prisinzano, L., Sanz-Forcada, J., Micela, G., et al. 2011, A\& A, 527, A77

Puzia, T. H., Perrett, K. M., \& Bridges, T. J. 2005, A\& $A$, 434, 909

Ramírez, I., Meléndez, J., \& Chanamé, J. 2012, ApJ, 757, 164

Ransom, S. M., Stairs, I. H., Archibald, A., et al. 2014, in American Astronomical Society Meeting Abstracts, Vol. 223, American Astronomical Society Meeting Abstracts, 140-144

Reid, M. J., Menten, K. M., Brunthaler, A., et al. 2014, ApJ, 783, 130

Rejkuba, M., Dubath, P., Minniti, D., \& Meylan, G. 2007, A\&AA, 469, 147

Renaud, F., Boily, C. M., Fleck, J.-J., Naab, T., \& Theis, C. 2008, MNRAS, 391, L98

Renaud, F. \& Gieles, M. 2013, MNRAS, 431, L83

Renzini, A. \& Fusi Pecci, F. 1988, ARA\&A, 26, 199

Reylé, C. \& Robin, A. C. 2002, A\& A, 384, 403

Richtler, T., Hilker, M., Kumar, B., et al. 2014, A\&A, 569, A41 
Richtler, T., Kumar, B., Bassino, L. P., Dirsch, B., \& Romanowsky, A. J. 2012, A\& A, 543, L7

Rivilla, V. M., Jiménez-Serra, I., Martín-Pintado, J., \& Sanz-Forcada, J. 2014, MNRAS, 437, 1561

Robitaille, T. P. \& Whitney, B. A. 2010, ApJ, 710, L11

Román-Zúñiga, C. G., Ybarra, J. E., Megías, G. D., et al. 2015, AJ, 150, 80

Rübke, K., Herrero, A., Borissova, J., et al. 2015, in Highlights of Spanish Astrophysics VIII, ed. A. J. Cenarro, F. Figueras, C. Hernández-Monteagudo, J. Trujillo Bueno, \& L. Valdivielso, 614-614

Russeil, D., Schneider, N., Anderson, L. D., et al. 2013, A\&A, 554, A42

Sadoun, R. \& Colin, J. 2012, MNRAS, 426, L51

Salinas, R., Jílková, L., Carraro, G., Catelan, M., \& Amigo, P. 2012, MNRAS, 421, 960

San Roman, I., Muñoz, C., Geisler, D., et al. 2015, A\&A, 579, A6

Sbordone, L., Bonifacio, P., Marconi, G., Buonanno, R., \& Zaggia, S. 2005, A\&A, 437, 905

Sbordone, L., Salaris, M., Weiss, A., \& Cassisi, S. 2011, A $\xi A$, 534, A9

Schaerer, D. \& Charbonnel, C. 2011, MNRAS, 413, 2297

Schmeja, S., Kharchenko, N. V., Piskunov, A. E., et al. 2014, A\&A, 568, A51

Scholz, R.-D., Kharchenko, N. V., Piskunov, A. E., Röser, S., \& Schilbach, E. 2015, A\&A, 581, A39

Schuberth, Y., Richtler, T., Hilker, M., et al. 2010, A\&A, 513, A52

Seth, A. C., van den Bosch, R., Mieske, S., et al. 2014, Nature, 513, 398

Shanahan, R. L. \& Gieles, M. 2015, MNRAS, 448, L94

Simpson, R. J., Povich, M. S., Kendrew, S., et al. 2012, MNRAS, 424, 2442

Simunovic, M. \& Puzia, T. H. 2014, ApJ, 782, 49

Skrutskie, M. F., Cutri, R. M., Stiening, R., et al. 2006, AJ, 131, 1163

Smith, R., Sánchez-Janssen, R., Fellhauer, M., et al. 2013, MNRAS, 429, 1066

Smolinski, J. P., Martell, S. L., Beers, T. C., \& Lee, Y. S. 2011, AJ, 142, 126

Soares, J. B., Bica, E., Ahumada, A. V., \& Clariá, J. J. 2008, A\&SA, 478, 419

Solin, O., Haikala, L., \& Ukkonen, E. 2014, A\&A, 562, A115

Solin, O., Ukkonen, E., \& Haikala, L. 2012, A\&A, 542, A3

Sollima, A., Martínez-Delgado, D., Valls-Gabaud, D., \& Peñarrubia, J. 2011, ApJ, 726, 47

Strader, J., Caldwell, N., \& Seth, A. C. 2011, AJ, 142, 8

Sung, H., Lim, B., Bessell, M. S., et al. 2013, Journal of Korean Astronomical Society, 46, 103

Tadross, A. L. 2008, MNRAS, 389, 285

Tadross, A. L. 2009, New A, 14, 200

Tadross, A. L. 2011, Journal of Korean Astronomical Society, 44, 1

Taylor, M. A., Puzia, T. H., Harris, G. L., et al. 2010, ApJ, 712, 1191

Thompson, B., Frinchaboy, P., Kinemuchi, K., Sarajedini, A., \& Cohen, R. 2014, AJ, 148, 85

Thompson, M. A., Urquhart, J. S., Moore, T. J. T., \& Morgan, L. K. 2012, MNRAS, 421, 408

Trenti, M., Padoan, P., \& Jimenez, R. 2015, ApJ, 808, L35

Tudorica, A., Georgiev, I. Y., \& Chies-Santos, A. L. 2015, A\&A, 581, A84

Turner, D. G., Majaess, D. J., Lane, D. J., et al. 2012, MNRAS, 422, 2501

Usher, C., Forbes, D. A., Brodie, J. P., et al. 2012, MNRAS, 426, 1475

Veljanoski, J., Mackey, A. D., Ferguson, A. M. N., et al. 2014, MNRAS, 442, 2929

Ventura, P. \& D'Antona, F. 2008, MNRAS, 385, 2034

Villanova, S., Geisler, D., Carraro, G., Moni Bidin, C., \& Muñoz, C. 2013, ApJ, 778, 186

Villanova, S., Piotto, G., \& Gratton, R. G. 2009, A\& A, 499, 755

Villegas, D., Jordán, A., Peng, E. W., et al. 2010, ApJ, 717, 603

West, M. J., Jordán, A., Blakeslee, J. P., et al. 2011, A $\& A$, 528, A115

Whitmore, B. C., Chandar, R., \& Fall, S. M. 2007, AJ, 133, 1067

Whitmore, B. C., Gilmore, D., Leitherer, C., et al. 2005, AJ, 130, 2104

Woodley, K. A., Gómez, M., Harris, W. E., Geisler, D., \& Harris, G. L. H. 2010a, AJ, 139, 1871

Woodley, K. A., Harris, W. E., Puzia, T. H., et al. 2010b, ApJ, 708, 1335

Wright, N. J., Parker, R. J., Goodwin, S. P., \& Drake, J. J. 2014, MNRAS, 438, 639

Wylie-de Boer, E., Freeman, K., Williams, M., et al. 2012, ApJ, 755, 35 
Yang, W., Meng, X., Bi, S., et al. 2011, ApJ, 731, L37

Yong, D., Grundahl, F., Johnson, J. A., \& Asplund, M. 2008, ApJ, 684, 1159

Zasowski, G., Beaton, R. L., Hamm, K. K., et al. 2013, AJ, 146, 64

Zhang, H.-X., Peng, E. W., Côté, P., et al. 2015, ApJ, 802, 30

Zhu, Q., Davies, B., Figer, D. F., \& Trombley, C. 2009, ApJ, 702, 929 